\title{
BMJ Open How does acupuncture affect insulin sensitivity in women with polycystic ovary syndrome and insulin resistance? Study protocol of a prospective pilot study
}

\author{
Yanhua Zheng, ${ }^{1,2}$ Elisabet Stener-Victorin, ${ }^{3}$ Ernest $\mathrm{H}$ Y Ng, ${ }^{4}$ Juan $\mathrm{Li},{ }^{1,5}$ \\ Xiaoke $\mathrm{Wu}^{6}{ }^{6}$ Hongxia $\mathrm{Ma}^{1,5}$
}

To cite: Zheng Y, StenerVictorin $\mathrm{E}, \mathrm{Ng} \mathrm{EHY}$, et al. How does acupuncture affect insulin sensitivity in women with polycystic ovary syndrome and insulin resistance? Study protocol of a prospective pilot study. BMJ Open 2015;5:e007757. doi:10.1136/bmjopen-2015007757

- Prepublication history for this paper is available online. To view these files please visit the journal online (http://dx.doi.org/10.1136/ bmjopen-2015-007757).

Received 23 January 2015 Revised 17 February 2015 Accepted 19 February 2015

CrossMark

For numbered affiliations see end of article.

Correspondence to Dr Hongxia Ma; doctorhongxia@126.com

\section{ABSTRACT}

Introduction: Hyperinsulinaemia and insulin resistance (IR) are key features of polycystic ovary syndrome (PCOS) and metabolic syndrome. The effect of 5 weeks of acupuncture treatment has been investigated in a completed prospective pilot trial (Clinicaltrials.gov: NCT01457209), and acupuncture with electrical stimulation applied to insulin-resistant rats with dihydrotestosteroneinduced PCOS was shown to improve insulin sensitivity. Therefore, we now aim to conduct a prospective pilot study to evaluate whether using the same acupuncture treatment protocol given over a longer period of time (6 months) than in the previous pilot trial will improve insulin sensitivity in women with PCOS and IR. Our hypothesis is that acupuncture with combined manual and lowfrequency electrical stimulation of the needles will improve insulin sensitivity in women with PCOS and IR.

Methods/analysis: This is a prospective pilot trial. A total of 112 women with PCOS and IR will be recruited and categorised according to their body mass index (BMI) as normal weight (BMI=18.5 $-23 \mathrm{~kg} / \mathrm{m}^{2}$ ) or as overweight/obese $\left(\mathrm{BMl}>23 \mathrm{~kg} / \mathrm{m}^{2}\right)$. Acupuncture will be applied three times per week for 6 months at $30 \mathrm{~min}$ per treatment. The primary outcome will be the change in insulin sensitivity before and after 6 months of acupuncture treatment, as measured by an oral glucose tolerance test.

Ethics/dissemination: Ethical approval of this study has been granted from the ethics committee of the First Affiliated Hospital of Guangzhou Medical University (No. 2013039). Written and informed consent will be obtained from each patient before any study procedure is performed, according to good clinical practice. The results of this trial will be disseminated in a peer-reviewed journal and presented at international congresses.

Trial registration numbers: NCT02026323 and ChiCTR-OCH-13003921.

\section{BACKGROUND}

Features of polycystic ovary syndrome (PCOS) include ovulatory dysfunction, clinical and biochemical signs of hyperandrogenism, and the presence of polycystic ovaries on pelvic scanning. ${ }^{12}$ PCOS is the most common endocrine and metabolic disorder in reproductiveage women, ${ }^{2}$ and is found in $5.6 \%$ of Chinese women aged 19-45 years, as shown in a recent community-based study. ${ }^{1}$ The main metabolic phenotype is hyperinsulinaemia and insulin resistance (IR), which is observed in about $50-70 \%$ of all women with PCOS. ${ }^{34}$ Importantly, IR can be present in lean and in obese women with PCOS, ${ }^{56}$ and especially in those with hyperandrogenism and ovulatory dysfunction. ${ }^{78}$

PCOS causes significant menstrual and fertility issues, but over the past few decades its additional metabolic and cardiovascular risks have become apparent. Women with PCOS display IR and reduced insulin responsiveness ${ }^{9}$ and have at least a threefold increased risk of developing type 2 diabetes mellitus. ${ }^{10}$ IR in women with PCOS has been attributed to postbinding defects in adipocytes and skeletal muscle insulin signalling. ${ }^{9} 11$ Furthermore, compensatory hyperinsulinaemia fuels ovarian androgen production by stimulating androgen production and secretion by theca cells, and by reducing sex hormone-binding globulin (SHBG) levels, which increases free androgens and further exacerbates PCOS symptoms. ${ }^{12}$ Thus, there is a strong association between hyperinsulinaemia and hyperandrogenaemia.

Overweight/obesity is a common feature of PCOS. ${ }^{13}$ A Korean study showed that $61 \%$ of women with PCOS were lean, $10.3 \%$ were overweight and $28.4 \%$ were obese. ${ }^{14}$ Obesity aggravates most of the metabolic 
dysfunctions in women with PCOS. Women with comorbid PCOS and depression have been found to have higher body mass index (BMI) and IR compared with women with PCOS without depression, ${ }^{15}$ and this increase in BMI negatively affects quality of life. ${ }^{16}$

Despite the high prevalence of IR, impaired glucose tolerance and/or type 2 diabetes mellitus in women with PCOS, there is no consensus on the best long-term management of these conditions. Pharmacological treatments, including metformin, are symptom oriented and usually effective but have unpleasant gastrointestinal side effects. Therefore, it is important to evaluate other nonpharmacological treatment strategies because most women with PCOS require long-term treatment.

Acupuncture, which is one of the main treatment modalities of traditional Chinese medicine, is increasingly being used in the area of reproductive endocrinology and infertility in many different parts of the world. Interestingly, increasing the treatment frequency and the number of treatments leads to higher ovulation frequency, ${ }^{17}$ indicating the importance of the correct treatment dose. In a secondary analysis of the trial by Jedel et al, ${ }^{18}$ low-frequency electroacupuncture (EA) decreased high plasminogen activator inhibitor 1 (PAI-1) activity without affecting insulin sensitivity, as measured by the euglycaemic hyperinsulinaemic clamp. ${ }^{19}$ Clearly, the intensity, frequency and duration of low-frequency EA treatment in that study were too low to affect insulin sensitivity. Support for this assumption comes from our experimental studies in the dihydrotestosterone (DHT)-induced PCOS rat model, in which we demonstrated that low-frequency EA improves whole-body insulin sensitivity in a dosedependent manner. ${ }^{2021}$

EA induces systemic as well as local effects involving intracellular insulin signalling pathways in skeletal muscle and adipose tissue. ${ }^{20} 22{ }^{23}$ Furthermore, EA has been shown to reduce plasma glucose levels by promoting insulin production and to improve insulin sensitivity by inducing secretion of endogenous $\beta$-endorphin in different rodent models of diabetes mellitus. ${ }^{24} 25$ The potential role of acupuncture in the treatment of women with PCOS and IR has not yet been determined, and this is an important area to investigate because most women with PCOS require long-term treatment. There is one completed prospective pilot trial investigating the effect of 5 weeks of acupuncture treatment with combined manual and low-frequency electrical stimulation of the needles (Clinicaltrials.gov: NCT01457209). Therefore, the aim of this pilot study is to investigate the clinical effectiveness of acupuncture on insulin sensitivity in women with PCOS and IR when acupuncture is given over a longer period of time (6 months) than in the previous pilot trial using the same treatment protocol. Our hypothesis is that acupuncture with combined manual and low-frequency electrical stimulation of the needles will improve insulin sensitivity in women with PCOS and IR.

\section{MATERIALS AND METHODS}

Study design

This is a single-centre prospective pilot study, and participants will be enrolled from the First Affiliated Hospital of Guangzhou Medical University. The trial has been registered at ClinicalTrial.gov (NCT02026323) and with the Chinese Clinical Trials Registry (ChiCTR-OCH-13003921).

\section{Participants}

Participants will be recruited if they fulfil all of the inclusion criteria and do not meet any of the exclusion criteria.

\section{Inclusion criteria}

1. Age between 18 and 40 years.

2. $B M I \geq 18.5 \mathrm{~kg} / \mathrm{m}^{2}$.

3. Presence of PCOS as defined by the Rotterdam criteria and including at least two of the following three features:

- Oligomenorrhoea or amenorrhoea: Oligomenorrhoea is defined as an intermenstrual interval $>35$ days or $<8$ menstrual bleedings in the past year. Amenorrhoea is defined as complete cessation of menstrual cycles for 6 months or more when a patient has previously had regular cycles and for 12 months or more when the patient has had irregular cycles.

- Clinical or biochemical hyperandrogenism: Biochemical hyperandrogenaemia is defined as a total serum testosterone concentration above $60 \mathrm{ng} /$ $\mathrm{dL},{ }^{26}$ and clinical hyperandrogenism is defined as a Ferriman-Gallwey (FG) score $\geq 5$, in mainland China. $^{27}$

- Polycystic ovary morphology: This is defined as $\geq 12$ antral follicles (2-9 $\mathrm{mm}$ in diameter) or an ovarian volume $>10 \mathrm{~mL}$ on transvaginal scanning. ${ }^{28}$

4. Presence of IR as defined by the homeostatic model assessment (HOMA-IR: (fasting insulin $(\mu \mathrm{U} / \mathrm{mL}) \times$ fasting glucose $(\mathrm{mmol} / \mathrm{L})) / 22.5)$. A value $\geq 2.14$ will be considered to be indicative of IR. ${ }^{29}$

5. No desire to bear children and having used barrier methods of contraception for 1 year.

6. Willingness to sign the consent form.

\section{Exclusion criteria}

1. Having other endocrine disorders such as hyperprolactinaemia (defined as two prolactin levels measured at least 1 week apart of $25 \mathrm{ng} / \mathrm{mL}$ or greater or as determined by local normative values), non-classic congenital adrenal hyperplasia (17-hydroxyprogesterone $<3 \mathrm{nmol} / \mathrm{L}$ ), or androgen-secreting tumours.

2. Follicle-stimulating hormone (FSH) levels $>15 \mathrm{mIU} / \mathrm{mL}$. A normal level within the last year is adequate for entry.

3. Uncorrected thyroid disease defined as thyroidstimulating hormone (TSH) $<0.2 \mathrm{mIU} / \mathrm{mL}$ or $\mathrm{TSH}>5.5 \mathrm{mIU} / \mathrm{mL}$. A normal level within the last year is adequate for entry. 
4. Patients with type I diabetes mellitus or type I and type II who are receiving antidiabetic medications such as insulin, thiazolidinediones, acarbose, sulfonylureas, or other medications that are likely to confound the effects of the study. Patients currently receiving metformin for a diagnosis of type I or type II diabetes or for PCOS are also specifically excluded.

5. Suspected Cushing's syndrome.

6. Use of hormones or other medications in the past 3 months, including Chinese herbal prescriptions, which might affect the outcome.

7. Pregnancy within the past 6 weeks.

8. Postabortion or post partum within the past 6 weeks.

9. Breast feeding within the past 6 months.

10. Receiving acupuncture treatment related to PCOS within the past 2 months.

11. Having undergone a bariatric surgery procedure within the past 12 months or being in a period of acute weight loss.

12. Having known congenital adrenal hyperplasia.

13. Lack of written consent to participate in the study. Eligible participants will be recruited and categorised according to their BMI as normal weight $(\mathrm{BMI}=18.5-$ $\left.23 \mathrm{~kg} / \mathrm{m}^{2}\right)$ or overweight/obese $\left(\mathrm{BMI} \geq 23 \mathrm{~kg} / \mathrm{m}^{2}\right) .{ }^{30}$

\section{Interventions}

The acupuncture protocol is based on Western medical theories, and the study protocol follows the CONSORT ${ }^{31}$ and STandards for Reporting Interventions in Clinical Trials of Acupuncture (STRICTA) ${ }^{32}$ recommendations with detailed descriptions of the treatment, including the number of needles used, depth of needle insertion, how needles will be stimulated (manual or electrical), frequency of sessions and length of the treatment period. Acupuncture will be given according to a fixed protocol by traditional Chinese medicine practitioners educated in theoretical and practical acupuncture. The protocol is based on experimental studies elucidating the effect of acupuncture in rodent models of diabetes mellitus and PCOS ${ }^{20-22}$ and in women with PCOS. ${ }^{19} 33$

\section{Acupuncture protocol}

Acupuncture will be given three times per week over 6 months for a total of 80 sessions. Disposable, sterilised needles for single use made of stainless steel $(0.20 \times 30 \mathrm{~mm}$ and $0.20 \times 40 / 50 \mathrm{~mm}$; Hwoto, Suzhou Medical Appliance Factory, Suzhou, China) will be inserted to a depth of 15$35 \mathrm{~mm}$ in segmental acupuncture points located in the abdominal and leg muscles, with innervations corresponding to the ovaries and pancreas that have been shown to improve insulin sensitivity in rodents. Two sets of acupuncture points will be alternated for every other treatment session. The two acupuncture protocols follow the STRICTA recommendations and are presented in table 1. The rationale for using the traditional nomenclature of acupuncture points is that they are well described in the literature, and acupuncturists trained according to medical acupuncture theories or trained in classic traditional Chinese medicine theories all know the locations of such points and how the needles should be inserted. Thus, using the acupuncture name/number makes it easier for all practitioners to know where the needles were placed. All needles will be stimulated manually by rotation until needle sensation is evoked, which is indicative of activation of a-delta and c-fibres when the needles are inserted. Needles placed in the abdominal muscles and leg muscles will be attached to an electrical stimulator (Export Abteilung, Schwa-Medico GmbH, Germany) and electrically stimulated at $2 \mathrm{~Hz}$ for $30 \mathrm{~min}$ during each treatment session. Needles not connected to the electrical stimulator will be stimulated manually four times during each $30 \mathrm{~min}$ treatment session (table 1).

\section{Study procedures}

This pilot study has two phases. The first phase is the 6 months of acupuncture treatment, and the second phase is 6 months of follow-up (figure 1). Each specific visit and measurement is summarised in table 2.

The women in the study will be screened for IR. Those with a HOMA-IR ((fasting insulin $(\mu \mathrm{U} / \mathrm{mL}) \times$ fasting glucose $(\mathrm{mmol} / \mathrm{L})) / 22.5) \geq 2.14$ will be included and divided into the normal weight (BMI $=18.5-23 \mathrm{~kg} / \mathrm{m}^{2}$ ) group and the overweight/obese (BMI $\geq 23 \mathrm{~kg} / \mathrm{m}^{2}$ ) group. After inclusion and baseline measurements, acupuncture treatment will start and last for 6 months followed by 6 months of follow-up.

\section{Outcome measurements \\ Primary outcome}

The primary outcome will be the changes in HOMA-IR between baseline and after 6 months of acupuncture treatment, and between baseline and the 6-month follow-up. The oral glucose tolerance test (OGTT) with $75 \mathrm{~g}$ glucose will be performed in all participants after an overnight fast. Blood samples will be obtained to measure plasma glucose and serum insulin at 0,60 and 120 min during the OGTT.

\section{Secondary outcomes}

1. HOMA-B: Islet $\beta$-cell function will be evaluated by the formula (20×fasting insulin $(\mathrm{mU} / \mathrm{mL}) /($ fasting plasma glucose $(\mathrm{mmol} / \mathrm{L})-3.5))^{34}$ and by the $\mathrm{C}$ peptide index (CPI), which is measured as (fasting $\mathrm{C}$ peptide $(\mathrm{nmol} / \mathrm{L}) /$ fasting plasma glucose $(\mathrm{mmol} / \mathrm{L}) \times 100){ }^{35}$

2. The insulin response to glucose will be assessed by calculating the area under the curve during the OGTT for glucose $\left(\mathrm{AUC}_{\mathrm{glu}}\right)$ and insulin $\left(\mathrm{AUC}_{\mathrm{ins}}\right)$ using the trapezoidal rule. ${ }^{36}$

3. Menstrual bleeding patterns will be evaluated.

4. Body composition will be determined as weight, height, waist-to-hip circumference and BMI.

5. Metabolic measures will include glycated haemoglobin, C peptide, adiponectin, fasting cholesterol, fasting triglycerides (TG), apolipoprotein A1 (ApoA1), apolipoprotein $\mathrm{B}(\mathrm{ApoB})$ and blood pressure. 


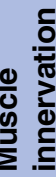

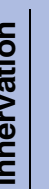

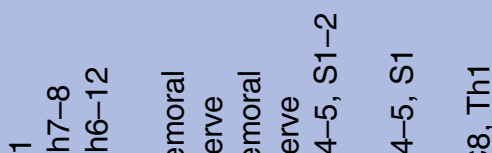

$\approx$

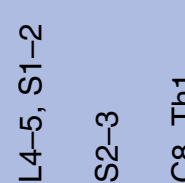

ᄃ

$\frac{\ddot{c}}{\tilde{c}}$

$\frac{\pi}{2} \frac{\pi}{\pi}$

क)

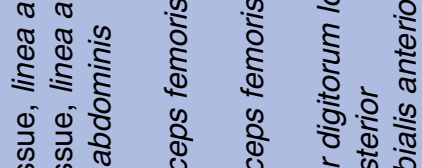

क्र

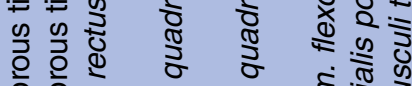

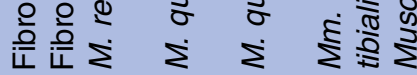

कิ

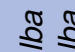

응

พ

फ़

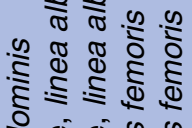

के की की

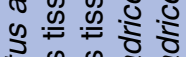

ป⿱艹

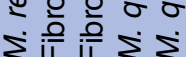

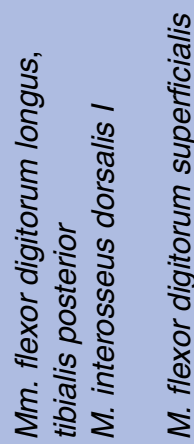

$\stackrel{Ð}{=}$

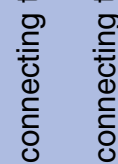

$\stackrel{\grave{\Phi}}{\leftrightarrows}$

흥

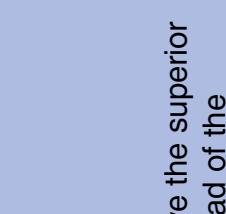

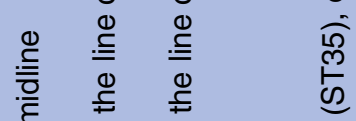

号

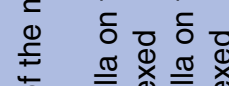

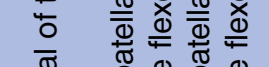

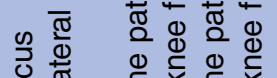

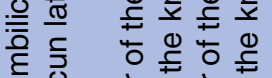

는

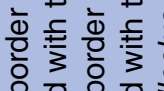

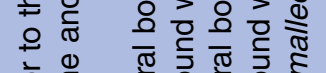

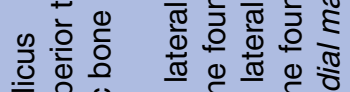

ปั

辛竞

రิ

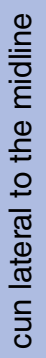

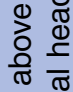

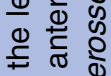

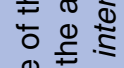

है क을

흥

은

今 $5 \frac{2}{0}$

के की के है

通

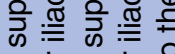

요웅

뜬 흔 옫흔

흥 응

흔

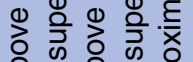

㐫 흥 을

तु

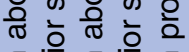

ब。

ชั

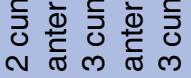

ठ흐응

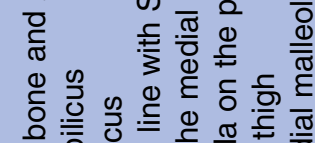

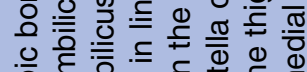

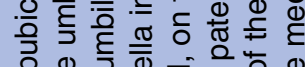

응

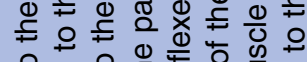

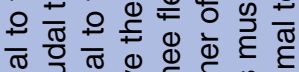

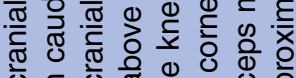

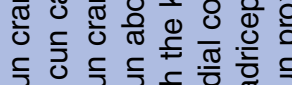

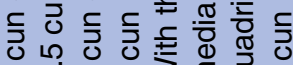

$\sum \Sigma$

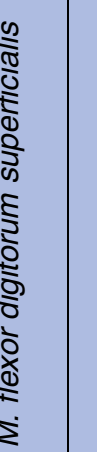

$\sum_{\substack{0 \\ \mathrm{O}}}^{\infty}$

蛋

등 


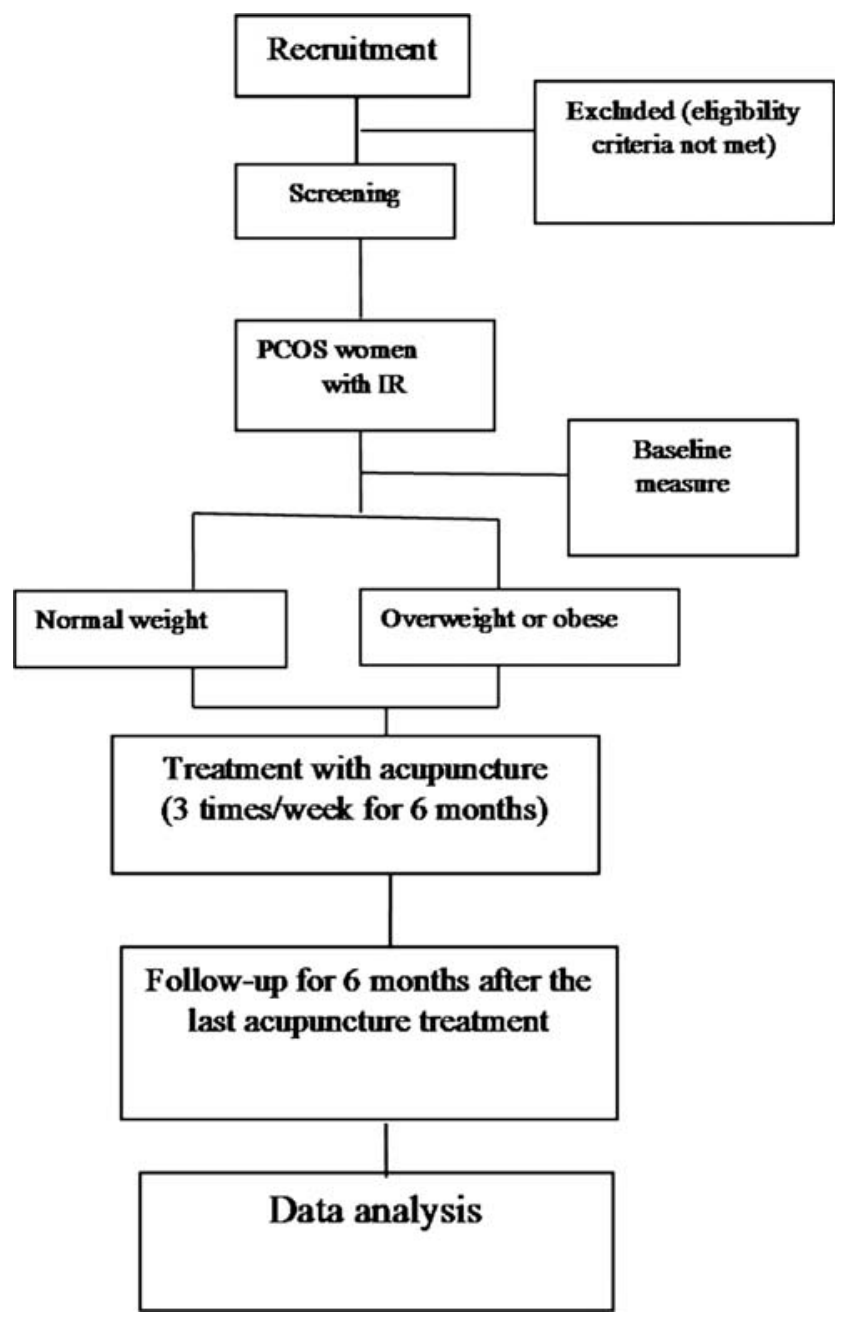

Figure 1 (IR, insulin resistance; PCOS, polycystic ovary syndrome).

6. Endocrine measures will include hirsutism as assessed by FG score, the presence of acne, and serum concentrations of testosterone, SHBG, FSH, luteinising hormone and dehydroepiandrosterone sulfate (DHEAS).

7. Questionnaires will include the short form-36 (SF36), ${ }^{37}$ the PCOS questionnaire (PCOSQ) ${ }^{38}$ generic and diagnosis-specific health-related quality of life questionnaires, and the Chinese Quality of Life (ChQOL) questionnaire. ${ }^{39}$ Symptoms of anxiety and depression will be assessed by the Zung Self-Rating Anxiety Scale (Zung SAS), and the Zung Self-Rating Depression Scale (Zung SDS) ${ }^{40}$ In addition, the International Physical Activity Questionnaire (IPAQ) ${ }^{41}$ written in Chinese will be used to measure the frequency and duration of moderate physical activity every week.

8. Adverse events will be recorded.

\section{Data entry and quality control of data}

Case report forms will be developed for data entry, and quality control of the data will be handled at two different levels. The investigators will be required to ensure the accuracy of the data as the first level of control, and the second level will include data monitoring and validation that will be carried out on a regular basis throughout the study.

\section{Sample size calculations and statistical analysis}

Our previous study on treating obese women with PCOS with abdominal acupuncture showed that the HOMA-IR was significantly reduced after treatment $(3.9 \pm 1.4)$ compared with baseline measurements $(2.5 \pm 1.7) .{ }^{33}$ A HOMA-IR $\geq 2.14$ was considered to be abnormal. ${ }^{29}$ If we assume a more moderate $20 \%$ reduction in HOMA-IR, that is, a reduction by 0.78 , we will have to recruit 40 overweight or obese participants and 40 normal-weight participants. With an estimated $40 \%$ drop out rate, we plan to recruit 56 normal weight and 56 overweight/obese women with PCOS.

One sample of the Kolmogorov-Smirnov test will be used to test the normal distribution of continuous

Table 2 Overview of study visits, including screening, baseline measurements, after-treatment measurements and follow-up measurements

\begin{tabular}{|c|c|c|c|c|c|c|c|c|}
\hline & \multirow{2}{*}{$\begin{array}{l}\text { Screening and } \\
\text { baseline visit }\end{array}$} & \multicolumn{6}{|c|}{ Month } & \multirow{2}{*}{$\begin{array}{l}\text { Follow-up } \\
\text { 6th month }\end{array}$} \\
\hline & & 1st & 2nd & 3rd & 4th & 5th & 6th & \\
\hline $\begin{array}{l}\text { Body composition (weight, height, waist circumference, hip } \\
\text { circumference) }\end{array}$ & $\sqrt{ }$ & $\sqrt{ }$ & $\sqrt{ }$ & $\sqrt{ }$ & $\sqrt{ }$ & $\sqrt{ }$ & $\sqrt{ }$ & $\sqrt{ }$ \\
\hline \multirow{2}{*}{$\begin{array}{l}\text { Menstrual cycle diary } \\
\text { Fasting blood samples for Apoa1, ApoB, TC, TG, C } \\
\text { peptide, HbA1c }\end{array}$} & $\sqrt{ }$ & $\sqrt{ }$ & $\sqrt{ }$ & $\sqrt{ }$ & $\sqrt{ }$ & $\sqrt{ }$ & $\sqrt{ }$ & $\sqrt{ }$ \\
\hline & $\sqrt{ }$ & & & & & & $\sqrt{ }$ & $\sqrt{ }$ \\
\hline $\begin{array}{l}\text { Fasting blood samples for FSH, LH, SHBG, T, E2, P, } \\
\text { DHEAS }\end{array}$ & $\sqrt{ }$ & & & & & & $\sqrt{ }$ & $\sqrt{ }$ \\
\hline Transvaginal ultrasound & $\sqrt{ }$ & & & & & & $\sqrt{ }$ & $\sqrt{ }$ \\
\hline Questionnaire & $\sqrt{ }$ & & & & & & $\sqrt{ }$ & $\sqrt{ }$ \\
\hline $\mathrm{FG} / \mathrm{acne}$ & $\sqrt{ }$ & $\sqrt{ }$ & $\sqrt{ }$ & $\sqrt{ }$ & $\sqrt{ }$ & $\sqrt{ }$ & $\sqrt{ }$ & $\sqrt{ }$ \\
\hline OGTT & $\sqrt{ }$ & & & $\sqrt{ }$ & & & $\sqrt{ }$ & $\sqrt{ }$ \\
\hline
\end{tabular}


variables. Continuous variables will be shown as means \pm SDs if they are normally distributed or as medians with IQRs if they are not normally distributed. Statistical comparisons will be carried out according to the intention to treat by Student $\mathrm{t}$ test, Mann-Whitney U-test, and Wilcoxon signed-ranks test for continuous variables and by $\chi^{2}$ tests for categorical variables where appropriate. All statistical analyses of the data will be performed using the SPSS program V.21.0 (SPSS Inc, Chicago, Illinois, USA), and a $\mathrm{p}$ value $<0.05$ will be considered statistically significant.

\section{Safety and ethical considerations}

Acupuncture is a safe procedure, and few side effects have been reported. The major risks of acupuncture are local skin irritation, discomfort and vasovagal reactions during the procedure. The women who agree to participate in the study will sign a consent form.

\section{DISCUSSION}

Acupuncture has been used in the treatment of women with PCOS, ${ }^{17-19} 33$ but the effect of acupuncture on IR in women with PCOS is still unknown. This prospective pilot study has been designed, therefore, to evaluate whether acupuncture improves insulin sensitivity in women with PCOS and IR. The research seeks to add significantly to the clinical evidence base and to allow conclusions to be made on the role of acupuncture in the treatment of PCOS.

A limitation of the present study is that it is a singlecentre study without comparison groups, and it can be argued that it lacks scientific rigour because of this. To increase the validity of the study, we will have experts controlling the quality of study procedure through regular site visits. The experts will monitor the recruitment of study participants, the measurement and treatment procedures, and the data analysis. We plan to conduct a randomised controlled trial at a later date using standard care as a control.

This pilot study was conceived and designed in 2013, and the first participant was recruited on 18 February 2014. As of 18 December 2014, out of 368 women approached, 176 did not fulfil selection criteria, 85 declined participation and 107 consented to participate in the study. In total, 99 women were screened and 83 were recruited.

\section{Author affiliations}

${ }^{1}$ Research Institute of Integrated Traditional Chinese Medicine and Western Medicine, Guangzhou Medical University, Guangzhou, China

${ }^{2}$ Department of Traditional Chinese Medicine, The Fourth Affiliated Hospital of Guangzhou Medical University, Guangzhou, China

${ }^{3}$ Department of Physiology and Pharmacology, Karolinska Institutet,

Stockholm, Sweden

${ }^{4}$ Department of Obstetrics and Gynaecology, The University of Hong Kong, Hong Kong Special Administrative Region, Hong Kong, Hong Kong

${ }^{5}$ Department of Traditional Chinese Medicine, The First Affiliated Hospital of Guangzhou Medical University, Guangzhou, China

${ }^{6}$ Department of Gynecology, First Affiliated Hospital National Key Discipline, Specialty and Trial Base Heilongjiang University of Chinese Medicine, China
Contributors YZ, ES-V and EHYN contributed equally to this work. ES-V, EHYN and HM conceived and designed the study. YZ, EHYN and ES-V drafted and critically revised the manuscript for important intellectual content. HM sought funding and ethical approval. All the authors contributed to the further writing of the manuscript and approved the final manuscript.

Funding This work was supported in part by the National Foundation of Natural Science of China (grant number 81273786), the Science and Technology Planning Project of Guangdong Province in China (grant number 2011B031700042) and funded by the national clinical trial base 'Optimization study of traditional Chinese medicine therapy for women with polycystic ovary syndrome' (grant number JDZX2012043).

Competing interests None declared.

Patient consent Obtained.

Ethics approval This study was approved by the ethics committee of the First Affiliated Hospital of Guangzhou Medical University (No. 2013039).

Provenance and peer review Not commissioned; externally peer reviewed.

Data sharing statement No additional date are available.

Open Access This is an Open Access article distributed in accordance with the Creative Commons Attribution Non Commercial (CC BY-NC 4.0) license, which permits others to distribute, remix, adapt, build upon this work noncommercially, and license their derivative works on different terms, provided the original work is properly cited and the use is non-commercial. See: http:// creativecommons.org/licenses/by-nc/4.0/

\section{REFERENCES}

1. Li R, Zhang Q, Yang D, et al. Prevalence of polycystic ovary syndrome in women in China: a large community-based study. Hum Reprod 2013;28:2562-9.

2. Norman RJ, Dewailly D, Legro RS, et al. Polycystic ovary syndrome. Lancet 2007;370:685-97.

3. Legro RS, Castracane VD, Kauffman RP. Detecting insulin resistance in polycystic ovary syndrome: purposes and pitfalls. Obstet Gynecol Surv 2004;59:141-54.

4. Yin Y, Hong-mei X, Guang-xiu L. Analysis of insulin resistance and metabolism characteristic of the Hans PCOS women. China J Mod Med 2007; 17:1625-30.

5. Chang RJ, Nakamura RM, Judd HL, et al. Insulin resistance in nonobese patients with polycystic ovarian disease. J Clin Endocrinol Metab 1983;57:356-9.

6. Stepto NK, Cassar S, Joham AE, et al. Women with polycystic ovary syndrome have intrinsic insulin resistance on euglycaemichyperinsulaemic clamp. Hum Reprod 2013;28:777-84.

7. Fauser BC, Tarlatzis BC, Rebar RW, et al. Consensus on women's health aspects of polycystic ovary syndrome (PCOS): the Amsterdam ESHRE/ASRM-Sponsored 3rd PCOS Consensus Workshop Group. Fertil Steril 2012;97:28-38.e25.

8. Moran L, Teede $\mathrm{H}$. Metabolic features of the reproductive phenotypes of polycystic ovary syndrome. Hum Reprod Update 2009;15:477-88.

9. Ciaraldi TP, Aroda V, Mudaliar S, et al. Polycystic ovary syndrome is associated with tissue-specific differences in insulin resistance. J Clin Endocrinol Metab 2009;94:157-63.

10. Ehrmann DA, Liljenquist DR, Kasza K, et al. Prevalence and predictors of the metabolic syndrome in women with polycystic ovary syndrome. J Clin Endocrinol Metab 2006;91:48-53.

11. Dunaif $A, W u X$, Lee $A$, et al. Defects in insulin receptor signaling in vivo in the polycystic ovary syndrome (PCOS). Am J Physiol Endocrinol Metab 2001;281:E392-99.

12. Corbould A. Effects of androgens on insulin action in women: is androgen excess a component of female metabolic syndrome? Diabetes Metab Res Rev 2008;24:520-32.

13. Alvarez-Blasco F, Botella-Carretero JI, San Millan JL, et al. Prevalence and characteristics of the polycystic ovary syndrome in overweight and obese women. Arch Intern Med 2006;166:2081-6.

14. Lee $H$, Oh JY, Sung YA, et al. The prevalence and risk factors for glucose intolerance in young Korean women with polycystic ovary syndrome. Endocrine 2009;36:326-32

15. Hollinrake E, Abreu A, Maifeld M, et al. Increased risk of depressive disorders in women with polycystic ovary syndrome. Fertil Steril 2007;87:1369-76. 
16. Jones GL, Balen AH, Ledger WL. Health-related quality of life in PCOS and related infertility: how can we assess this? Hum Fertil (Camb) 2008;11:173-85.

17. Johansson J, Redman L, Veldhuis PP, et al. Acupuncture for ovulation induction in polycystic ovary syndrome: a randomized controlled trial. Am J Physiol Endocrinol Metab 2013;304: E934-43.

18. Jedel E, Labrie F, Oden A, et al. Impact of electro-acupuncture and physical exercise on hyperandrogenism and oligo/amenorrhea in women with polycystic ovary syndrome: a randomized controlled trial. Am J Physiol Endocrinol Metab 2011;300:E37-45.

19. Stener-Victorin E, Baghaei F, Holm G, et al. Effects of acupuncture and exercise on insulin sensitivity, adipose tissue characteristics, and markers of coagulation and fibrinolysis in women with polycystic ovary syndrome: secondary analyses of a randomized controlled trial. Fertil Steril 2012;97:501-8.

20. Johansson J, Yi F, Shao R, et al. intense acupuncture normalizes insulin sensitivity, increases muscle GLUT4 content, and improves lipid profile in a rat model of polycystic ovary syndrome. $\mathrm{Am} \mathrm{J}$ Physiol Endocrinol Metab 2010;299:E551-9.

21. Manneras L, Jonsdottir IH, Holmang A, et al. Low-frequency electro-acupuncture and physical exercise improve metabolic disturbances and modulate gene expression in adipose tissue in rats with dihydrotestosterone-induced polycystic ovary syndrome. Endocrinology 2008;149:3559-68.

22. Liang $F$, Chen R, Nakagawa A, et al. Low-frequency electroacupuncture improves insulin sensitivity in obese diabetic mice through activation of SIRT1/PGC-1 $\alpha$ in skeletal muscle. Evid Based Complement Alternat Med 2011;2011:735297.

23. Johansson J, Manneras-Holm L, Shao R, et al. Electrical vs manual acupuncture stimulation in a rat model of polycystic ovary syndrome: different effects on muscle and fat tissue insulin signaling. PLOS ONE 2013;8:e54357.

24. Chang SL, Lin JG, Chi TC, et al. An insulin-dependent hypoglycaemia induced by electroacupuncture at the Zhongwan (CV12) acupoint in diabetic rats. Diabetologia 1999;42:250-5.

25. Chang S-L, Lin K-J, Lin R-T, et al. Enhanced insulin sensitivity using electroacupuncture on bilateral Zusanli acupoints (ST 36) in rats. Life Sci 2006;79:967-71.

26. Shi Y, Guo M, Yan J, et al. Analysis of clinical characteristics in large-scale Chinese women with polycystic ovary syndrome. Neuro Endocrinol Lett 2007;28:807-10.

27. Zhao X, Ni R, Li L, et al. Defining hirsutism in Chinese women: a cross-sectional study. Fertil Steril 2011;96:792-6.

28. Balen AH, Laven JSE, Tan S-L, et al. Ultrasound assessment of the polycystic ovary: international consensus definitions. Hum Reprod Update 2003;9:505-14.
29. Chen X, Yang D, Li L, et al. Abnormal glucose tolerance in Chinese women with polycystic ovary syndrome. Hum Reprod 2006;21:2027-32.

30. Misra A, Chowbey P, Makkar BM, et al. Consensus statement for diagnosis of obesity, abdominal obesity and the metabolic syndrome for Asian Indians and recommendations for physical activity, medical and surgical management. J Assoc Physicians India 2009;57:163-70.

31. Moher D, Schulz KF, Altman D. The CONSORT Statement: revised recommendations for improving the quality of reports of parallelgroup randomized trials 2001. Explore (NY) 2005;1:40-5.

32. MacPherson H, Altman DG, Hammerschlag R, et al. Revised STandards for Reporting Interventions in Clinical Trials of Acupuncture (STRICTA): extending the CONSORT statement. PLoS Med 2010;7:e1000261.

33. Lai MH, Ma HX, Yao H, et al. [Effect of abdominal acupuncture therapy on the endocrine and metabolism in obesity-type polycystic ovarian syndrome patients]. Zhen Ci Yan Jiu 2010;35:298-302.

34. Matthews DR, Hosker JP, Rudenski AS, et al. Homeostasis model assessment: insulin resistance and beta-cell function from fasting plasma glucose and insulin concentrations in man. Diabetologia 1985;28:412-19.

35. Iwata M, Maeda S, Kamura Y, et al. Genetic risk score constructed using 14 susceptibility alleles for type 2 diabetes is associated with the early onset of diabetes and may predict the future requirement of insulin injections among Japanese individuals. Diabetes Care 2012;35:1763-70.

36. Kahn SE, Prigeon RL, McCulloch DK, et al. Quantification of the relationship between insulin sensitivity and beta-cell function in human subjects. Evidence for a hyperbolic function. Diabetes 1993;42:1663-72

37. McHorney CA, Ware JE Jr, Lu JF, et al. The MOS 36-item Short-Form Health Survey (SF-36): III. Tests of data quality, scaling assumptions, and reliability across diverse patient groups. Med Care 1994;32:40-66.

38. Cronin L, Guyatt G, Griffith L, et al. Development of a healthrelated quality-of-life questionnaire (PCOSQ) for women with polycystic ovary syndrome (PCOS). J Clin Endocrinol Metab 1998;83:1976-87.

39. Leung KF, Liu FB, Zhao L, et al. Development and validation of the Chinese Quality of Life Instrument. Health Qual Life Outcomes 2005;3:26

40. Marone J, Lubin B. Relationship between set 2 of the Depression adjective check lists (DACL) and Zung self-rating depression scale (SDS). Psychol Rep 1968;22:333-4.

41. Craig CL, Marshall AL, Sjostrom M, et al. International physical activity questionnaire: 12 -country reliability and validity. Med Sci Sports Exerc 2003;35:1381-95. 